\title{
Learning Impairment and Cholinergic Deafferentation after Cortical Nerve Growth Factor Deprivation
}

\author{
Humberto Gutiérrez, María Isabel Miranda, and Federico Bermúdez-Rattoni \\ Departamento de Neurociencias, Instituto de Fisiología Celular, Universidad Nacional Autónoma de México, 04510 \\ Mexico D. F., Mexico
}

\begin{abstract}
Cholinergic basal forebrain (CBF) neurons have been shown to respond in vivo to exogenous administration of NGF. Although neurotrophins and their receptors are widely expressed in the CNS, little data exist for the physiological significance of endogenous neurotrophin signaling in CNS neurons. To test directly whether cortically derived NGF is functionally required for the cholinergic functions mediated by the cerebral cortex, repeated injections of anti-NGF mAbs were locally applied into the insular cortex (IC) of rats. The biochemical results, using an in vivo microdialysis technique, showed a dramatic lack of extracellular release of acetylcholine after high potassium stimulation compared with controls. Furthermore, by using small injections of the neurotracer fluorogold, we found a corresponding disruption in the connectivity between the IC and the
\end{abstract}

CBF. Behavioral experiments showed that the NGF antibodies applied into the IC produced a significant disruption on the acquisition of conditioned taste aversion and inhibitory avoidance learning. However, the same animals were able to recall the taste aversion when the conditioning trial was established before injections of NGF antibodies. Given these results, it seems that cortical cholinergic functions are actively dependent on locally derived NGF in the adult normal brain, and that the cholinergic activity from the CBF is not necessary for recalling aversive stimuli, but is necessary for the acquisition of aversively motivated conditionings.

Key words: inhibitory avoidance; conditioned taste aversion; anti-NGF antibodies; memory; cholinergic basal forebrain; cortical cholinergic activity
The cholinergic basal forebrain $(\mathrm{CBF})$ complex provides widespread, topologically organized afferent cholinergic innervation to many brain regions, including the neocortex and hippocampus (Bigl et al., 1982). A number of studies have implicated these cholinergic neurons in the mediation of learning and memory processes in experimental animals (Etherington et al., 1987; Everitt et al., 1987; Dunnett and Fibiger, 1993; Nabeshima, 1993; Sinden et al., 1995). The role of NGF as a target-derived neurotrophic agent for certain cell populations in the peripheral nervous system has been well documented (Levi-Montalccini, 1987). This trophic concept has been extended to the CNS, and recent evidence suggests that NGF may be essential for supporting the cholinergic neurons of the basal forebrain (Araujo et al., 1990; Vazquez and Ebendal, 1991). The CBF neurons have also been demonstrated to be the major population of cells in the CNS expressing receptors for NGF (Vazquez and Ebendal, 1991; Urschel and Hulsebosch, 1992; Holtzman et al., 1995;). However, no direct evidence has shown an active, physiological dependency of the brain cholinergic neurons on a target-derived trophic factor (Mannes et al., 1994).

According to the neurotrophic hypothesis, NGF is synthesized by target cells of basal forebrain neuronal projections (i.e., neocortex, hippocampus, amygdala, and other brain structures), binds to NGF receptors located on nerve terminals, and is retrogradely

Received Nov. 18, 1996; revised Feb. 14, 1997; accepted Feb. 21, 1997.

This research was supported by DGAPA-UNAM Grant IN201893 and CONACyT Grants 9207-1554 and 3260P-N9608. We acknowledge the assistance of Oreste Carbajal and Federico Jandete, and we thank Christopher E. Ormsby Jenkins for revision and Yolanda Díaz de Castro for preparation of this manuscript.

Correspondence should be addressed to Federico Bermúdez-Rattoni, Instituto de Fisiología Celular, Universidad Nacional Autónoma de México, Apartado Postal 70-253, 04510 Mexico D. F., Mexico.

Copyright (C) 1997 Society for Neuroscience $\quad 0270-6474 / 97 / 173796-\bullet \$ 05.00 / 0$ transported to cell bodies of the basal forebrain neurons (Araujo et al., 1990; Cuello et al., 1992). Nonetheless, the massive ablation of cortical neurons and the presumed corresponding reduction in target-derived trophic support do not seem to have any impact on the viability of the CBF neurons (Minger and Davies, 1992). Furthermore, mutant mice lacking the NGF gene have been shown to develop basal forebrain cholinergic neurons in a normal fashion (Crowly et al., 1994), thus questioning the widely accepted dependency of mature $\mathrm{CBF}$ neurons on the availability of a target-derived neurotrophic factor (Minger and Davies, 1992). Although extensive cell death of both sympathetic and sensory neurons occurs after peripheral administration of antibodies against NGF at any stage of development, no similar effect has been found for the case of the central cholinergic neurons (Springer and Loy, 1985; Mannes et al., 1994;). Recently, however, it has been reported that chronic infusion of anti-NGF antibodies applied directly into the medial septum produced a significant decrease of the choline acetyltransferase activity in the hippocampus and significant disruption on the acquisition of an aversive learning task in normal adult rats (Nita et al., 1993).

The insular cortex (IC) of the rat is defined as an area spanning from the lateral frontal cortex to the perirhinal cortex in the rostrocaudal direction and from the ventral edge of the somatomotor cortex to the pyriform cortex in the dorsoventral direction (Saper, 1982). The IC has been related to the cognitive processing involved in the acquisition and retrieval of conditioned taste aversion (CTA) and inhibitory avoidance (IA). The CTA is a very robust and widely used model for the study of learning and memory processes (Garcia, 1990). In this behavioral model, an animal acquires aversion to a taste when it is followed by digestive malaise. The anatomical substrates responsible for CTA learning have been well established (Kiefer, 1985). In this regard, it has 


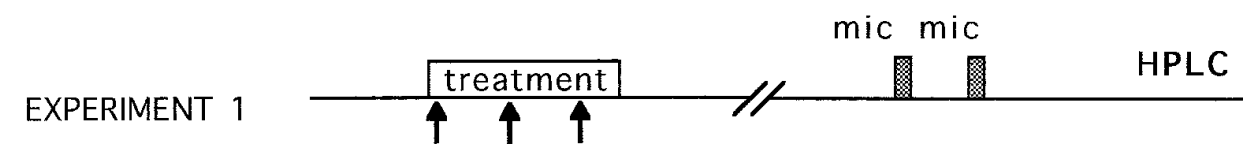

\begin{abstract}
Figure 1. Schematic representation of the experimental protocols used in this study. Arrows represent individual intracortical applications of anti-NGF, anti$\mathrm{IgG}$, or vehicle injections. Each box represents a particular relevant manipulation: mic, microdialysis procedure; $F G$, intracortical fluorogold injections; CTA Ac1, first CTA acquisition trial; Test1, first CTA retention test; $C T A A c 2$, second CTA acquisition trial; Test 2 , second CTA retention test; $I A$ ac, IA acquisition trial; $L A$ test, IA retention test.
\end{abstract}

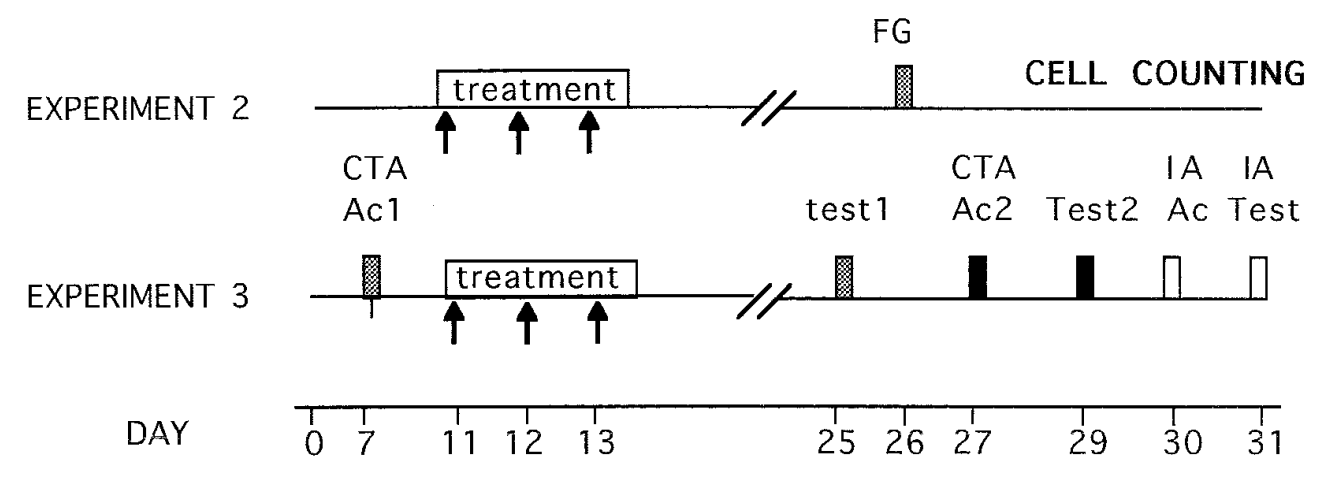

been shown that bilateral a lesion of the IC disrupts acquisition and retention of CTA (Kiefer and Brown, 1979; Yamamoto et al., 1980; Aggleton et al., 1981; Braun et al., 1982). Because IC lesions do not produce obvious deficits in gustatory or gastrointestinal sensibility, it has been suggested that IC lesions impair the mnemonic representation of tastes and their postgastrointestinal consequences (malaise; Kiefer, 1985). Furthermore, lesion experiments have also demonstrated an involvement of the IC in the acquisition and consolidation of spatial and IA learning tasks using either reversible or NMDA-induced lesions (BermúdezRattoni and McGaugh, 1991; Bermúdez-Rattoni et al., 1991).

We have shown previously that the adult IC is able to release significant amounts of radioactive acetylcholine (ACh) after high potassium depolarization and possesses choline acetyltransferase and cholinesterase activities (López-García et al., 1990). Moreover, excitotoxic lesions of the nucleus basalis magnocellularis (NBM) have implied an involvement of the ascending cholinergic pathway in the ability to learn CTA and IA (López-García et al., 1993). The IC thus offers an appropriate model for the analysis of the possible functional role of cortical NGF in the mature rat brain.

The following experiments were designed to reveal whether the cholinergic function of the $\mathrm{CBF}$ neurons projecting to the IC in the adult rat would be actively dependent on a target-derived trophic support (i.e., NGF dependency) in normal physiological situations, by investigating the biochemical, histological, and behavioral effects of repeated microinjections of anti-NGF mAbs directly into the IC.

\section{EXPERIMENT 1}

In this experiment, we used the in vivo microdialysis technique to study the biochemical effects of repeated infusions of anti-NGF mAbs through a cannula directed to the IC of adult rats. Animals were bilaterally implanted with microdialysis intracerebral guides directed to the IC. Each subject received repeated unilateral injections of anti-NGF, whereas the contralateral hemisphere received the corresponding vehicle control treatment. Comparisons of high potassium-stimulated ACh release between the antiNGF-treated and the contralateral cortex were made to obtain a quantitative measure of the cholinergic effect of the treatment.

\section{Materials and Methods}

Male Wistar rats weighing 250-300 gm were used in all the experiments. They were individually caged at $25^{\circ} \mathrm{C}$ in a $12 \mathrm{hr}$ light/dark cycle. All behavioral and biochemical manipulations were carried out in the light cycle.

General surgical procedure. On day 0 (see Fig. 1), bilateral (five animals) and unilateral (10 animals) implantation of microdialysis intracerebral guides directed to the IC were performed using the following procedure. Animals were anesthetized with sodium pentobarbital $(65 \mathrm{mg} / \mathrm{kg})$ and placed in a head holder, and guide cannulae [CMA12; Bioanalytical System (BAS)] were stereotaxically implanted with the following coordinates: anteroposterior, $+1.2 \mathrm{~mm}$ from bregma; lateral, $\pm 5.5 \mathrm{~mm}$; and dorsoventral, $-3.0 \mathrm{~mm}$. The microdialysis guides were attached firmly to the skull with dental acrylic cement and anchored with three stainless steel screws on the skull.

After a recovery period, on day 11, the group of animals with bilateral cannulae received repeated unilateral injections of $\mathrm{mAbs}$ to mouse NGF- $\beta$ (2.5 and 7 S; Boehringer Mannheim, Mannheim, Germany), whereas the contralateral hemisphere received the corresponding vehicle treatment. To rule out possible nonspecific effects of antibody infusions into the cortex, one-half of the unilaterally implanted group received repeated injections of polyclonal anti-IgG antibodies (Sigma, St. Louis, MO; $n=5)$. The other half of the unilaterally implanted group was kept as an intact control group $(n=5)$. For all groups, microinjections were made through the intracerebral guide using dental needles (30 gauge, which protrude $2.5 \mathrm{~mm}$ from the tip of the guide cannula) attached to a microinfusion pump (Carnegie Medicin). A total volume of $1 \mu \mathrm{l}$ per hemisphere of either PBS (vehicle), anti-IgG $(1 \mu \mathrm{g} / \mu \mathrm{l})$, or anti-NGF was infused with a flux rate of $0.07 \mu \mathrm{l} / \mathrm{min}$ during a $15 \mathrm{~min}$ period each day (during $3 \mathrm{~d}$ ). The intracortical infusions were given daily to nonrestrained conscious animals.

Dialysis was started on day 26 by connecting the microdialysis probe (BAS CMA12; diameter, $0.5 \mathrm{~mm}$; length, $3 \mathrm{~mm}$ ) to the intracerebral guide via microdialysis tubing (BAS FEP; $0.66 \mathrm{~mm}$ outer diameter, 0.12 $\mathrm{mm}$ inner diameter) to a microinfusion pump (Carnegie Medicin). This method allowed the sampling of an area wider than the target IC region. Ringer's solution containing neostigmine bromide (Sigma; $10 \mu \mathrm{M}$ ) was continuously perfused at a rate of $2 \mu \mathrm{l} / \mathrm{min}$. The first $60 \mathrm{~min}$ sampling was discarded, and then samples were collected every 15 min (i.e., $30 \mu \mathrm{l} /$ sample) and immediately frozen. Six samples were obtained for each analyzed hemisphere with a perfusion medium containing only the Ringer's solution. In the third sample, $\mathrm{KCl}(56 \mathrm{~mm})$ was added to the perfusion medium, whereas the $\mathrm{NaCl}$ concentration was lowered to 82 $\mathrm{mM}$ to maintain physiological osmolarity.

Analysis of ACh levels. On day 31, the samples were assayed for ACh levels using HPLC with electrochemical detection, using a mobile phase, $\mathrm{pH} 8.5$, containing $50 \mathrm{~mm}$ sodium phosphate buffer and $0.5 \%$ Kathon reagent (BAS) microbicide. Samples were injected on a polymeric reversed phase column (BAS ACh-choline assay kit). ACh and choline were then converted into hydrogen peroxide and betaine in a postcolumn enzyme reactor containing immobilized acetylcholinesterase and choline oxidase (BAS). The hydrogen peroxide was detected electrochemically with a platinum electrode set at $500 \mathrm{mV}$ (vs $\mathrm{Ag} / \mathrm{AgCl}$ ). The detection limit, defined as the amount of ACh producing a peak twice the basal noise, was approximately $0.2 \mathrm{pmol}$. 


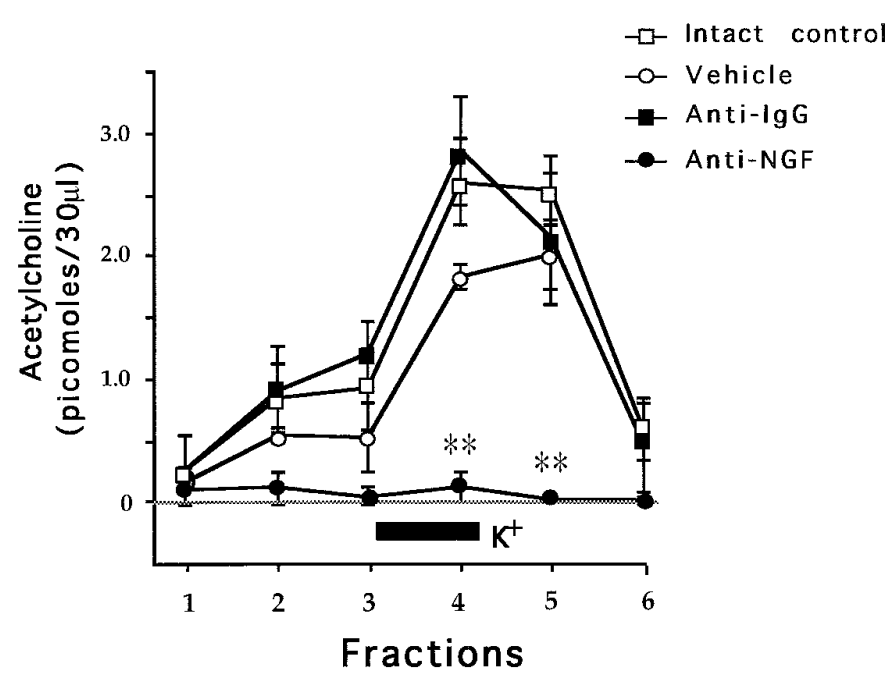

Figure 2. Effects of $56 \mathrm{~mm} \mathrm{KCl} \mathrm{(black} \mathrm{bar)} \mathrm{added} \mathrm{to} \mathrm{the} \mathrm{perfusion}$ medium on mean values $( \pm$ SEM $)$ of extracellular ACh release during the microdialysis procedure. ${ }^{* *} p<0.01$ compared against intact control. Note the lack of ACh release in the anti-NGF-treated hemispheres. The rest of the groups showed normal release of $\mathrm{ACh}$ after $\mathrm{KCl}$ stimulation.

One day after microdialysis, the rats were killed with an overdose of pentobarbital and transfused transcardially with a $4 \%$ solution of paraformaldehyde in phosphate buffer $(0.15 \mathrm{M}, \mathrm{pH} 7.4)$. The brains were placed overnight in paraformaldehyde and then transferred to a $20 \%$ buffered sucrose solution and stored at $4^{\circ} \mathrm{C}$ until they were cut. Serial coronal sections were cut in a freezing microtome at $40 \mu \mathrm{m}$ thickness, collected in PBS, and processed for visualization for cresyl violet staining after the standard procedure and with acetylcholinesterase histochemistry that was carried out on single mounted sections according to a modified protocol from Paxinos and Watson (1982). Briefly, the slides were incubated overnight in $50 \mathrm{~mm}$ sodium acetate buffer, $\mathrm{pH} 5.0,4 \mathrm{~mm}$ copper sulfate, $16 \mathrm{~mm}$ glicine, $4 \mathrm{~mm}$ acetylthiocholine iodide, and $0.1 \mathrm{~mm}$ ethopropazine. After incubation, the slides were immersed into a developing solution (1\% sodium sulfide, $\mathrm{pH} 7.5)$ for $10 \mathrm{~min}$.

\section{Results}

Figure 2 shows the time course of ACh release along $15 \mathrm{~min}$ fractions. Simple ANOVAs were used for comparison of ACh levels among groups and post hoc pairwise Fisher test where appropriate. During the first three fractions there were nonsignificant differences among groups $\left[F_{(3,16)}=0.2,2.17\right.$, and $4.3 ; p>$ 0.05]. After the addition of $56 \mathrm{mM} \mathrm{KCl}$ to the neostigminecontaining perfusion fluid during the third fraction, in the fourth and fifth fractions there were significant differences among groups $\left[F_{(3,16)}=17.91\right.$ and $\left.11.45 ; p<0.01\right]$. That is, the addition of $\mathrm{KCl}$ provoked an almost immediate overall increase in the ACh levels in both the vehicle-treated hemispheres and the anti-IgG control group, showing a normal pattern of cortical ACh release compared with the intact control group. The group treated with the anti-NGF showed a dramatic lack of cortical cholinergic release in fractions 4 and 5, even after high $\mathrm{KCl}$ stimulation, when compared with the intact control vehicle and anti-IgG groups $(p<0.01)$. In the sixth fraction, all groups showed similar cholinergic levels without any significant differences $\left[F_{(2,12)}=3.7 ; p>0.05\right]$.

Figure 3 shows the effect of anti-NGF treatment on histochemical staining for acetylcholinesterase activity. The extent of cholinergic depletion, because of application of anti-NGF antibodies, was evident as a marked decrease in the cortical acetylcholinesterase staining as compared with the control (vehicle) hemisphere. It is noteworthy that although a slight mechanical damage was apparent in the cortical parenchyma of the vehicle-treated hemi-

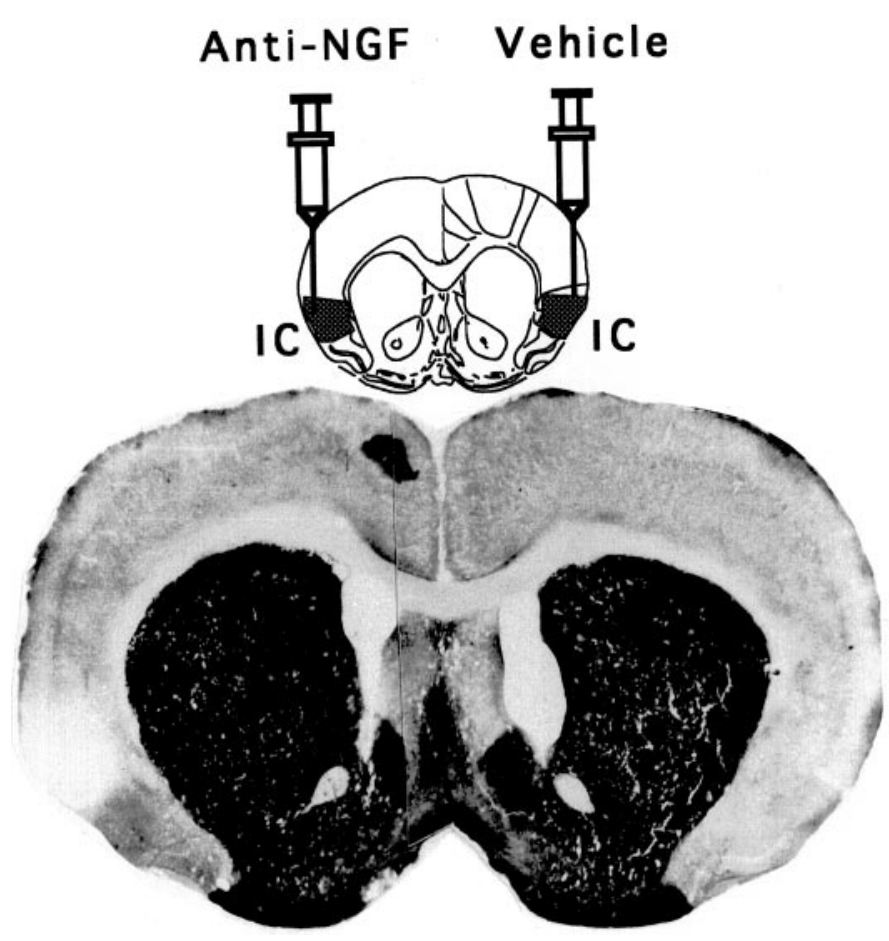

Figure 3. Schematic representation of repeated injections of anti-NGF $\mathrm{mAbs}$ into the IC, whereas the contralateral cortex received the corresponding vehicle treatment. Photomicrography of a coronal section showing the weak reaction for the AchE histochemistry in the anti-NGFtreated area (left) compared with the control side (right).

sphere, the measured levels of ACh were not significantly affected. Subsequent Nissl staining confirmed the localized presence of picnotic cells along the tract of the needle in both the anti-NGF and vehicle-treated control hemisphere (not shown).

\section{EXPERIMENT 2}

We have hypothesized that if cholinergic afferent fibers of cortical areas are actively dependent on target-derived trophic support, then a local anti-NGF treatment procedure should lead to a measurable reduction in connectivity between CBF (the presumed NGF-dependent system) and the treated cortical area. To test this hypothesis, a separate group of animals received unilateral injections of anti-NGF mAbs directly into the insular cortex, whereas the contralateral hemisphere received only vehicle injections. Twelve days later, each animal received bilateral injections of the fluorescent retrograde tracer fluorogold (FG) into the IC for comparisons of the number of FG-labeled cells in the basal forebrain and the ventroposteromedial thalamic nuclei, which is the thalamic relay of the IC, between the control and experimental hemispheres.

\section{Materials and Methods}

Following the same procedure described in Experiment 1, five animals received bilateral and another five received unilateral implantations of stainless steel cannulae (inner diameter, $0.33 \mathrm{~mm}$; outer diameter, 0.63 $\mathrm{mm}$; Small Parts, Inc.) directed to the same coordinates of the IC. The group of animals with bilateral cannulae were given in one side injections of anti-NGF mAbs, whereas the contralateral hemisphere received only vehicle injections (as described in Experiment 1). The unilaterally implanted group received the corresponding treatment of control anti-IgG polyclonal antibodies.

Fifteen days later, the fluorescent tracer FG (fluorochrome, 2.5\%) was carefully microinjected either bilaterally or unilaterally into the IC. To obtain samples of normal FG labeling in the basal forebrain, a third group 
of five intact animals received unilateral stereotaxic injections of the neurotracer and were kept as an intact control group. FG was dissolved in PBS, pH 7.0-7.4, back-filled into a 30 gauge needle that was attached via a Teflon tube (30 gauge; Small Parts, Inc.) to a $10 \mu \mathrm{l}$ syringe (Hamilton Co., Reno, NV), and injected in a total volume of $0.3 \mu$ l during $4.5 \mathrm{~min}$ at a rate of $0.07 \mu \mathrm{l} / \mathrm{min}$ with the use of an automated Sage syringe pump (Orion Research, Inc., Boston, MA). The needle was kept in place for 2 min after the injection to maximize diffusion of the FG into the IC. Four days later, animals were perfused, and $40 \mu \mathrm{m}$ sections were obtained. The injection site was verified histologically by locating the needle tract. The presence of FG in retrogradely labeled neurons in the basal forebrain and the posteroventromedial thalamus was studied with the aid of a fluorescence microscope. To determine the relative density of labeled neurons in these two nuclei, 12 sections throughout each structure were selected. These sections were approximately $80 \mu \mathrm{m}$ apart. Within a defined area of $0.125 \mathrm{~mm}^{2}$ for the posteroventromedial thalamus and 0.5 $\mathrm{mm}^{2}$ for the basal forebrain (NBM) in the region of highest cell density, labeled neurons were counted. For each section, four individual density measurements were made and then compared with the contralateral hemisphere, and the average number of labeled neurons among sections was determined for each brain.

\section{Results}

In control animals, the FG-labeled cells were found in the CBF, ipsilateral to the injection site almost exclusively in the nucleus basalis along the ventral and medial border of the globus pallidus, although some labeled cells were also seen in substancia innominata (see Fig. 4).

Furthermore, because the IC includes the gustatory neocortex, which receives afferent connections from the thalamic taste area (in the ventromedial nucleus of the thalamus), a great number of labeled neurons were found in this nucleus (See Fig. 4). Simple ANOVA was used for comparison of FG-labeled cell density among groups, and a post hoc Fisher test was used for comparisons between treated and control hemispheres. As shown in Figure $5 A$, there were no statistical differences in average density of labeled cells among groups in the corresponding thalamic area $\left[F_{(3,16)}=1.3 ; p>0.05\right]$. Simple ANOVA made on the labeled cells of the NBM showed significant differences among groups $\left[F_{(3,16)}=29.49 ; p<0.01\right]$. The injection side of anti-NGF showed a clear reduction of labeled cells compared with the intact, vehicle, and anti-IgG groups $(p<0.01$; Fig. $5 B)$. Both the vehicletreated side and the anti-IgG control group showed normal cell labeling as compared with the intact control.

\section{EXPERIMENT 3}

As long as the CFB input to the cortex is actively dependent on cortically derived NGF, and given the well known involvement of the ascending cholinergic pathway in cortically mediated learning processes, we were interested in determining whether local injections of anti-NGF antibodies would lead to a measurable deficit in the ability to learn CTA and IA. To test this hypothesis, two groups of animals received either repeated bilateral injections of anti-NGF antibodies or vehicle injections directly into the IC, whereas a third group of animals remained unoperated during the whole procedure as an intact control. Figure 1 (Experiment 3) shows a schematic representation of the general protocol used in this behavioral study. The first CTA acquisition (CTA Ac1) was established to determine whether the animals are able to recall (test 1) the taste aversion when conditioned before the application of the antibodies. The second acquisition trial (CTA Ac2) was established to determine the effect of the anti-NGF treatment on the ability to acquire CTA mediated by the IC. Each CTA can be induced independently by using different taste stimuli (i.e., saccharine and $\mathrm{NaCl}$ solutions). Because of the known involvement of the IC in the acquisition and consolidation of the IA learning task, we were also interested in determining the possible effects of repeated cortical injections of anti-NGF antibodies in this behavioral paradigm.

\section{Materials and Methods}

CTA. On day 0 (Fig. 1), 16 animals were bilaterally implanted with stainless steel cannulae directed to the insular cortex as described in General surgical procedure of Experiment 1. After recovery, on day 5 the animals were deprived of water for $24 \mathrm{hr}$ and were habituated to get their daily water intake for $15 \mathrm{~min}$ intervals each day (baseline): distilled water was given to the rat in graded test tubes for $15 \mathrm{~min}$ each day, and the consumption was recorded; this procedure was used for all rats during the $5 \mathrm{~d}$ before the training for CTA. On the acquisition day (day 7, according to Fig. 1), $0.1 \mathrm{M} \mathrm{NaCl}$ was given instead of water as a novel taste (CTA Ac1). Thirty min after drinking, rats received an i.p. injection of $0.4 \mathrm{M}$ $\mathrm{LiCl}$ to induce gastric malaise.

During the treatment day (day 11), animals were randomly divided in two groups: one group $(n=8)$ received repeated bilateral microinjections of anti-NGF; a second group received the corresponding bilateral vehicle treatment $(n=8)$. The microinjections were done as described in General surgical procedure in Experiment 1. In addition, a third group of animals $(n=8)$ was also trained and kept as an intact control group. On day 25 , after reestablishment of baseline consumption, water was again substituted by the $\mathrm{NaCl}$ solution to test CTA retention, and the amount of fluid intake was measured as an aversion index. On day 27, a second training procedure was repeated as before using $0.1 \%$ sodium saccharine as a novel taste (CTA Ac2). This new CTA acquisition was tested (test 2) on day 29 by again presenting the saccharine solution and measuring the amount of fluid intake.

$I A$. This task was carried out $1 \mathrm{~d}$ after the second retrieval test of CTA (day 31; Fig. 1). The apparatus consisted in a trough-shaped alley $(91 \mathrm{~cm}$ long, $20 \mathrm{~cm}$ wide at the top, $6.4 \mathrm{~cm}$ wide at the floor, and $15 \mathrm{~cm}$ deep) that was divided into two compartments, one illuminated $(31 \mathrm{~cm}$ long) and one dark ( $60 \mathrm{~cm}$ long). The floor of the dark compartment had a metal plate through which electric shocks were delivered. The two compartments were separated by a sliding door that retracted into the floor. On the acquisition trial each rat was placed in the illuminated compartment during $20 \mathrm{sec}$. After opening the door, as soon as the rat entered the dark compartment, the door was closed, and an unavoidable foot shock (1 $\mathrm{mA} / 2 \mathrm{sec}$ ) was delivered. On the retention test, $24 \mathrm{hr}$ after the acquisition trial, the rats were again placed in the illuminated compartment, and the time before entry into the dark compartment was recorded as the latency period. The total time spent in the light compartment (safe compartment) during a 10 min test period was recorded as the dwelling time. For each group both the latency period and the dwelling scores are shown as the median value ( \pm interquartile range).

\section{Results}

\section{$C T A$}

Simple ANOVA was done on the test day consumption volume for all groups. No differences were found in the baseline water intake among groups. As shown in Figure $6 \mathrm{~A}$, during the test presentation of $\mathrm{NaCl}$ (test 1), all of the groups showed reduced consumption, indicating that no disruption of taste aversion was detected in any of the tested groups $\left[F_{(2,22)}=0.03\right]$. In contrast, during the test presentation of saccharine after the post-treatment acquisition (Fig. 6B), a significant group difference was found $\left[F_{(2,22)}=6.56 ; p<0.01\right]$. A post hoc pairwise Fisher test showed that the anti-NGF-treated group had a significant disruption in the acquisition of taste aversion as indicated by the increased saccharine consumption when compared with the control and vehicle groups $(p<0.01)$ on the retention trial (test 2$)$.

\section{$I A$}

Nonparametric Kruskal-Wallis and post hoc Mann-Whitney $U$ test statistics were used on latency and dwelling scores for comparisons between groups. As shown in Figure $7 A$, there were differences in the median latency periods for the entrance into the dark chamber among groups $(\mathrm{H}=18.0 ; p<0.01)$ during the test trial (IA test). As expected, both the intact and the vehicle-treated 

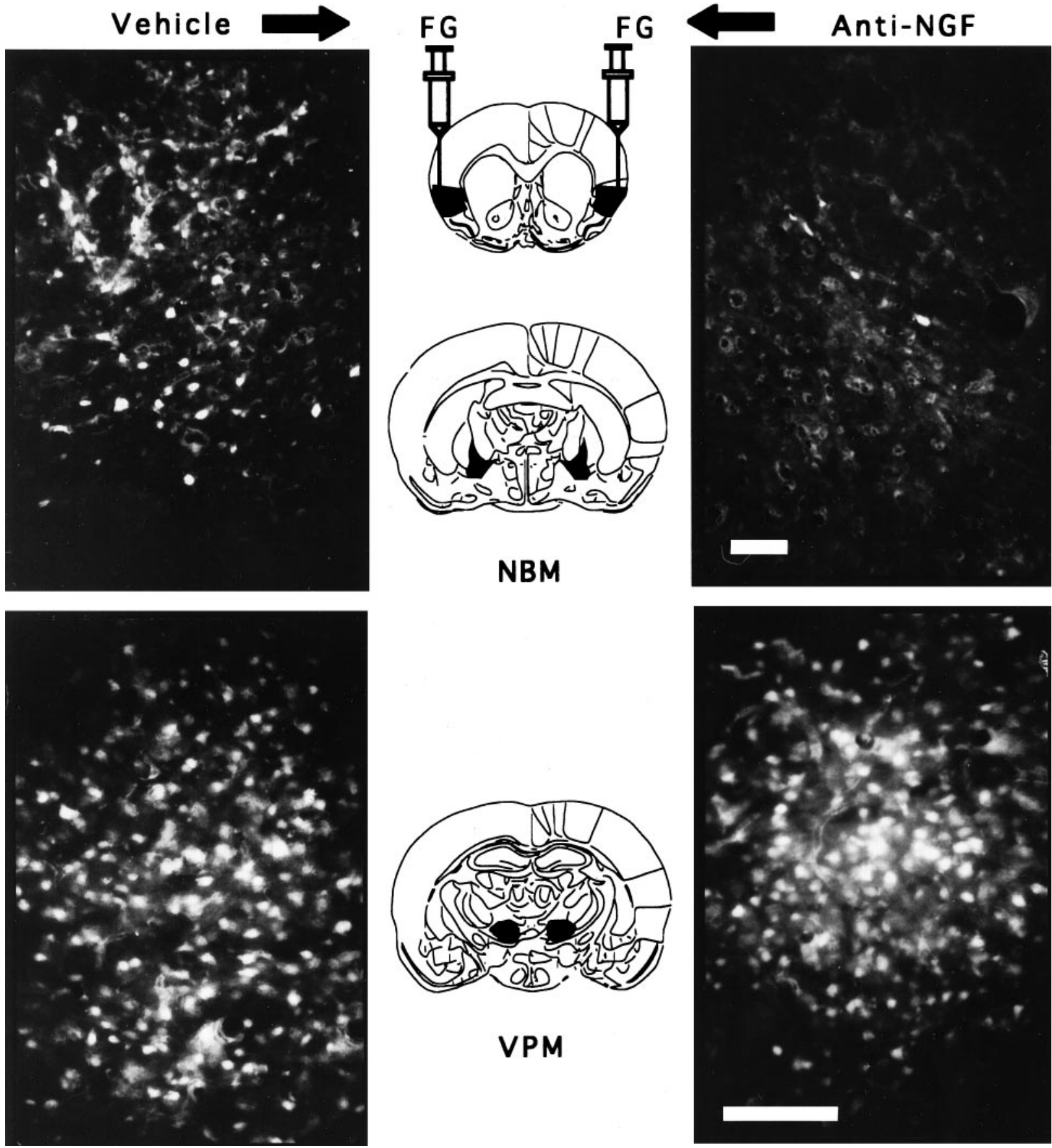

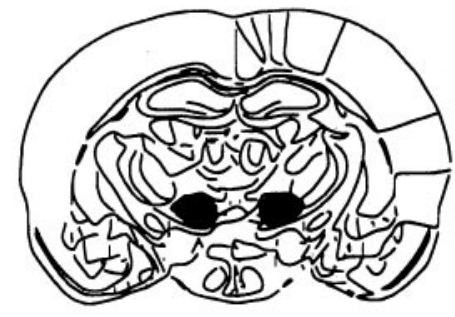

VPM

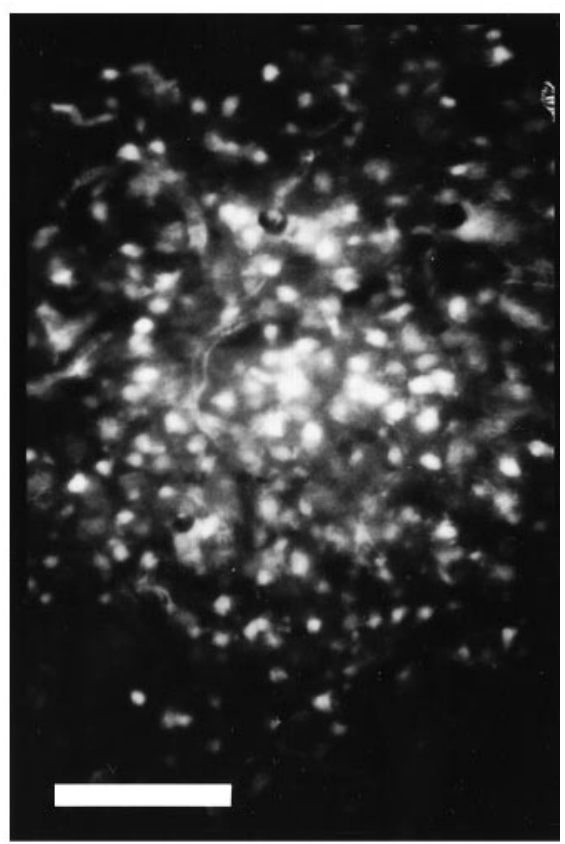

Figure 4. Photomicrographs showing FG labeling of NBM cells projecting to the IC after either intracortical vehicle injections (top left) or anti-NGF injections (top right). Labeling of posteroventromedial thalamus (VPM) cells projecting to the IC of the same brain: thalamic cells ipsilateral to the vehicle-treated cortex (bottom left) and cell labeling in the thalamus ipsilateral to the anti-NGF-treated cortex (bottom right). The four photomicrographs were obtained from the same brain. Scale bar, $100 \mu \mathrm{m}$.

group showed a high latency, whereas anti-NGF treatment caused a significant decrease in the latency period during the retention test. This result was closely paralleled by the corresponding dwelling scores (Fig. $7 B$ ). Statistical analysis showed significant differences among groups $(\mathrm{H}=18.7 ; p<0.01)$. The dwelling time for the anti-NGF-treated group was significantly lower than those of the vehicle-treated group and the intact control group $(p<0.01)$. These results indicate that anti-NGF-treated group showed disrupted ability to acquire IA learning.

\section{DISCUSSION}

In the present experiments, we have demonstrated that direct cortical infusion of anti-NGF mAbs results in a dramatic decrease in the local extracellular levels of $\mathrm{ACh}$ as determined by the in vivo microdialysis technique. Because the $\mathrm{CBF}$ ascending pathway constitutes by far the major cholinergic input to the neocortex (Bigl et al., 1982), it is likely that precisely this afferent pathway is being affected by the anti-NGF treatment. Using small injections of the neurotracer FG, we have found a corresponding disruption 

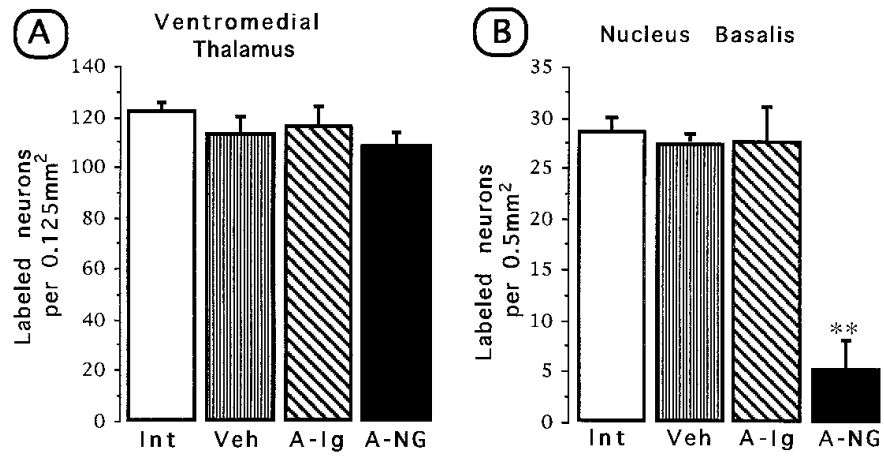

Figure 5. Quantitative FG retrograde tracing of thalamic cells projecting to the IC $(A)$ and forebrain cells projecting to the IC $(B)$ in the anti-NGFtreated side and the contralateral vehicle-treated side. Int, Intact control; $V e h$, vehicle; $A$-Ig, anti-IgG; $A-N G$, anti-NGF. Comparisons were made against the intact control group. ${ }^{* *} p<0.01$.
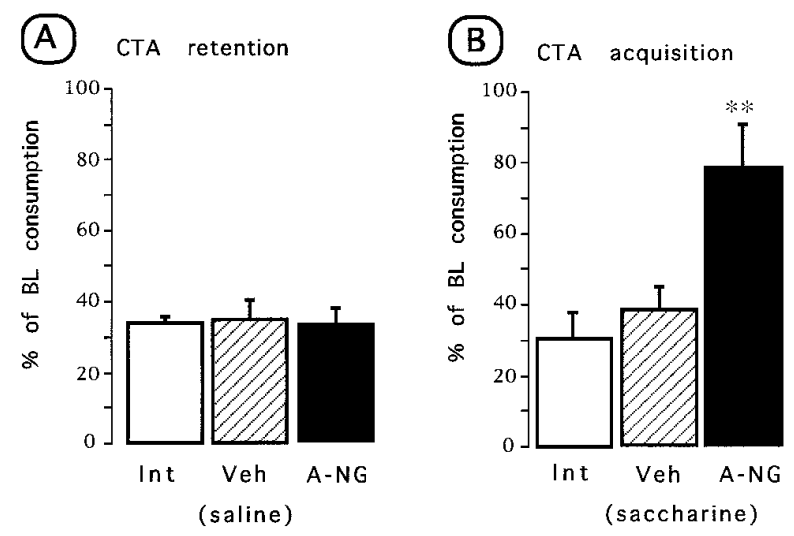

Figure 6. Effects of repeated anti-NGF or vehicle injections on CTA learning test. Aversion index expressed as mean $( \pm$ SEM) percentage of baseline consumption during the test trial. $A$, Retention of a previously acquired conditioning. $B$, Test of a post-treatment CTA acquisition. Int, Intact control; Veh, vehicle; $A-N G$, anti-NGF. ${ }^{* *} p<0.05$ versus intact control.

in connectivity between the IC and the basal complex ipsilateral to the anti-NGF-treated parenchyma, whereas the control side showed normal amounts of labeled cells. This effect in connectivity seems to be restricted to the basal forebrain, because the thalamic projections remained intact in both the contralateral control and the anti-NGF-treated side.

These results are in agreement with the observation that the CBF neurons constitute the major population of cells expressing receptors for NGF (Vazquez and Ebendal, 1991; Urschel and Hulsebosch, 1992; Holtzman et al., 1995;). Further evidence that these neurons are able to respond to exogenously added NGF has come from studies of fimbria fornix transection in rats and from nonlesioned, aged animals (Hefti, 1986; Ogawa et al., 1993; Fisher, 1994; Lindsay et al., 1994). Although neurotrophins and their receptors are widely expressed in the $\mathrm{CNS}$, relatively little data exist for the physiological role of neurotrophin signaling in CNS neurons. It is to be noted that simply documenting expression of a neurotrophic factor and its receptor does not clarify its actual functional significance. To determine the neurotrophic importance of NGF to the cortical cholinergic input, we suppressed biologically the local endogenous levels of NGF, with specific mAbs to NGF, which has been proven to be a useful approach (Nita et al., 1993; Van-der-Zee et al., 1995). Although

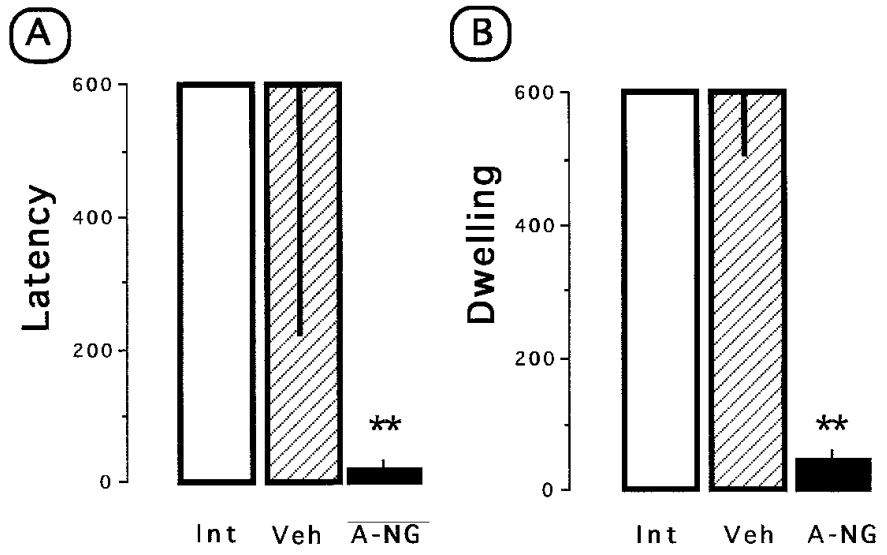

Figure 7. Effect of repeated anti-NGF or vehicle injections on IA learning test. $A$, Median ( \pm IQ range) latency to enter the dark compartment. $B$, Total time spent in the safe compartment during the $600 \mathrm{sec}$ test period. Int, Intact control; $V e h$, vehicle; $A-N G$, anti-NGF. ${ }^{*} p<0.01$ versus intact control.

administration of anti-NGF antiserum in fetal rats has been shown to reduce cholinergic neuron populations in the septum hippocampus and nucleus basalis (Vantini et al., 1989), no such an effect has been found when the treatment occurs in the adult animal (Springer and Loy, 1985; Mannes et al., 1994). However, recently it has been reported that chronic infusion of anti-NGF antibodies directly into the medial septum results in a significant decrease of choline acetyltransferase activity in the hippocampus (Nita et al., 1993). Our biochemical and histological results suggest that cholinergic input, as well as the physical connection between the cortex and the $\mathrm{CBF}$, is actively dependent on cortically derived NGF. This study thus provides support to the notion that the CBF function is physiologically dependent on a target (cortically)-derived NGF in the adult normal brain. However, whether the survival of the basal cholinergic neurons is in fact dependent on a target-derived neurotrophic factor, as stated by the neurotrophic hypothesis in its classical formulation, remains an open question.

As mentioned, bilateral lesions of IC disrupt acquisition and retention of CTA (Benjamin and Pfaffman, 1955; Kiefer, 1985). Furthermore, it has been demonstrated that the IC is also involved in the acquisition and consolidation of spatial and IA learning tasks (Bermúdez-Rattoni et al., 1991; Bermúdez-Rattoni and McGaugh, 1991). On the other hand, excitotoxic lesions of the nucleus basalis magnocellularis have been shown to impair acquisition and retrieval of CTA, as well as a number of learned behaviors including IA task (Etherington et al., 1987; Everitt et al., 1987; López-García et al., 1993). It should be pointed out that no effects on CTA conditioning after NBM lesions have been reported (Tardif, 1990; Kesner et al., 1992), although differential effects of NBM lesions on cortical cholinergic markers have been found depending on the excitotoxin used (Dunnet et al., 1991). Nevertheless, given the observed depletion of cholinergic activity, our behavioral results are in agreement with the well documented involvement of the ascending cholinergic pathway in cortically mediated learning processes (Fitzgerald and Burton, 1983; Etherington et al., 1987; Everitt et al., 1987; Sinden et al., 1995). In our behavioral study, anti-NGF treatment resulted in a marked impairment in the acquisition of both CTA and IA learning.

However, the role of the cortical cholinergic activity in the information retrieval mechanisms is less well known. Our re- 
sults suggest a cholinergic independent process during memory retrieval. That is, despite the biochemical and histological effects after anti-NGF treatment, there were no differences between control and anti-NGF-treated animals in the CTA retrieval test (evocation). This result clearly suggests that cortical NGF-dependent cholinergic activity is not required during information retrieval in this behavioral paradigm. Our data are in agreement with a previous study in which we found that IC-lesioned animals that received either cholinergically rich or cholinergically poor grafts were both able to recall a previously learned CTA. However, only those animals receiving cholinergically rich tissue were able to learn a novel CTA (Ormsby and Bermúdez-Rattoni, 1995). In addition, we have been able to demonstrate that supplementing IC fetal grafts with NGF not only induces recovery of cognitive function but also brings cholinergic activity back to normal unoperated control levels, as measured by in vivo and in vitro biochemical analyses (Escobar et al., 1993; Russell et al., 1994).

In this regard, it has been proposed recently that the cholinergic NBM cortical projection could play an important role only in the early stages of learning (Durkin and Toumane, 1992). Direct support for this view has been provided by previous observations that the post-test time duration of cholinergic activation became progressively shorter as a function of repeated daily training and the associated gain in task mastery. This decline in temporal involvement may ultimately reach such a degree that the intervention of central cholinergic neurons may no longer be functionally required during memory retrieval (Durkin and Toumane, 1992). Analogous observations have been found in terms of posttraining vulnerability to scopolamine-induced amnesia of spatial reference memory in mice (Toumane and Durkin, 1993).

The results of this study extend previous work, which investigated the effect of anti-NGF treatment in the CNS, and provide support to the notion that the CBF function is actively and physiologically dependent on a target (cortically)-derived NGF in the adult normal brain. Furthermore, they suggest that the cholinergic activity from the basal forebrain is not necessary for recalling aversive stimuli but is necessary for the acquisition of aversively motivated conditionings.

\section{REFERENCES}

Aggleton JP, Petrides M, Iversen SD (1981) Differential effects of amygdaloid lesion on conditioned taste aversion learning by rats. Physiol Behav 27:397-403.

Araujo DM, Chabot JG, Quirion R (1990) Potential neurotrophic factors in the mammalian nervous system: functional significance in the developing and aging brain. Int Rev Neurobiol 32:141-174.

Benjamin RM, Pfaffman C (1955) Cortical localization of taste in the albino rat. J Neurophysiol 18:56-64.

Bermúdez-Rattoni F, Intrioni-Collinson IB, McGaugh JL (1991) Reversible inactivation of the insular cortex by tetrodotoxin produces retrograde and anterograde amnesia for inhibitory avoidance and spatial learning. Proc Natl Acad Sci USA 88:5379-5382.

Bermúdez-Rattoni F, McGaugh JL (1991) Insular cortex and amygdala lesions differentially affect acquisition on inhibitory avoidance and conditioning taste aversion. Brain Res 49:165-170.

Bigl V, Woolf NJ, Butcher LL (1982) Cholinergic projection from the basal forebrain to frontal, parietal, temporal, occipital, and cingulate cortices: a combined fluorescent tracer and acetylcholinesterase analysis. Brain Res Bull 8:727-749.

Braun JJ, Lasiter PS, Kiefer SW (1982) The gustatory neocortex of the rat. Physiol Psychol 10:13-45.

Crowly C, Spencer SD, Nishimura MC, Chen KS, Pitts-Meek S, Armanini MP, Ling LH, McMahon SB, Shelton DL, Levinson AD, Phillips HS (1994) Mice lacking nerve growth factor display perinatal loss of sen- sory and sympathetic neurons yet develop basal forebrain cholinergic neurons. Cell 76:1001-1011.

Cuello AC, Maysinger D, Garofalo L (1992) Trophic factor effects on cholinergic innervation in the cerebral cortex of adult rat brain. Mol Neurobiol 6:451-461.

Dunnett SB, Everitt BJ, Robbins TW (1991) The basal forebrain cortical cholinergic system: interpreting the functional consequences of excitotoxic lesions. Trends Neurosci 14:494-501.

Dunnett SB, Fibiger HC (1993) Role of forebrain cholinergic system in learning and memory: relevance to cognitive deficits of aging and Alzheimer's dementia. Progr Brain Res 98:413-420.

Durkin TP, Toumane A (1992) Septo-hippocampal and nBM-cortical cholinergic neurons exhibits differential time-courses of activation as a function of both type and duration of spatial memory testing in mice. Behav Brain Res 50:43-52.

Escobar ML, Jiménez N, López-Garcia JC, Tapia R, Bermúdez-Rattoni F (1993) Nerve growth factor with insular cortical grafts induces recovery of learning and reestablishes graft choline acetyltransferase activity. J Neural Transplant Plast 4:167-172.

Etherington RE, Mittleman G, Robbins TW (1987) Comparative effects of nucleus basalis and fimbria-fornix lesions on delayed matching and alteration tests memory. Neurosci Res Commun 22:441-469.

Everitt BJ, Robbins TW, Evenden JL, Marston HM, Jones GH, Sirkia TE (1987) The effects of excitotoxic lesions of the substancia innominata, ventral and dorsal globus pallidus on the acquisition and retention of a conditional visual discrimination: implication for cholinergic hypothesis of learning and memory. Neuroscience 22:441-469.

Fisher W (1994) Nerve growth factor reverses spatial memory impairments in aged rats. Neurochem Int 25:47-52.

Fitzgerald RE, Burton MJ (1983) Neophobia and conditioned taste aversion deficits in the rat produced by undercutting temporal cortex. Physiol Behav 30:203-206.

Garcia J (1990) Learning without memory. J Cogn Neurosci 2:287-305.

Hefti F (1986) Nerve growth factor promotes survival of septal cholinergic neurons after fimbrial transections. J Neurosci 6:2155-2162.

Holtzman DM, Kilbridge J, Li Y, Cunningham ET, Lenn NJ, Clary DO, Reichardt LF, Mobley WC (1995) TrkA expression in the CNS: evidence for the existence of several novel NGF-responsive CNS neurons. J Neurosci 15:1567-1576.

Kesner RP, Berman RF, Tardif R (1992) Place and taste aversion learning: role of basal forebrain, parietal cortex and amygdala. Brain Res Bull 29:345-353.

Kiefer SW (1985) Neural mediation of conditioned food aversions. Ann NY Acad Sci 443:100-109.

Kiefer SW, Brown JJ (1979) Acquisition of taste avoidance habits in rats lacking gustatory neocortex. Physiol Psychol 7:245-250.

Levi-Montalccini R (1987) The nerve growth factor 35 years later. Science 237:1154-1162.

Lindsay RM, Wiegand SJ, Altar CA, DiStefano PS (1994) Neurotrophic factors: from molecule to man. Trends Neurosci 17:182-190.

López-García JC, Bermúdez-Rattoni F, Tapia R (1990) Release of acetylcholine, $\gamma$-aminobutyrate, dopamine and glutamate, and activity of some related enzymes, in rat gustatory neocortex. Brain Res 523:100-104.

López-García JC, Fernandez-Ruiz J, Escobar ML, Bermúdez-Rattoni F, Tapia R (1993) Effects of excitotoxic lesions of the nucleus basalis magnocellularis on conditioned taste aversion and inhibitory avoidance in the rat. Pharmacol Biochem Behav 45:147-152.

Mannes LM, Kastin AJ, Weber J, Banks WA, Beckman BS, Sadina JE (1994) The neurotrophins and their receptors: structure, function and neuropathology. Neurosci Behav Rev 18:143-159.

Minger SL, Davies P (1992) Persistent innervation of the rat neocortex by basal forebrain cholinergic neurons despite the massive reduction of cortical target neurons. Exp Neurol 117:139-150.

Nabeshima T (1993) Bahavioral aspects of cholinergic transmission: role of basal forebrain cholinergic system in learning and memory. Prog Brain Res 98:405-411.

Nita A, Murase M, Furukawa Y, Hayashi K, Hasegawa T, Nabeshima T (1993) Memory impairment and neural dysfunction after continuous infusion of anti-nerve growth factor antibody into the septum in adult rats. Neuroscience 57:495-499.

Ogawa S, Nabeshima T, Kameyama T, Hayashi K (1993) Effects of nerve growth factor (NGF) in rats with basal forebrain lesions. Jpn J Pharmacol 61:141-144. 
Ormsby C, Bermúdez-Rattoni F (1995) Induced remembrance of taste aversions by cortical implants. Soc Neurosci Abstr 20:1211.

Paxinos G, Watson C (1986) The rat brain in stereotaxic coordinates. San Diego: Academic.

Russell RW, Escobar ML, Booth RA, Bermúdez-Rattoni F (1994) Accelerating behavioral recovery after cortical lesions. II. In vivo evidence for cholinergic involvement. Behav Neural Biol 61:81-92.

Saper CB (1982) Convergence of autonomic and limbic projections in the insular cortex of the rat. J Comp Neurol 2:163-173.

Sinden JD, Hodges H, Gray JA (1995) Neural transplantation and recovery of cognitive function. Behav Brain Sci 18:10-35.

Springer JE, Loy R (1985) Intrahippocampal injection of antiserum to nerve growth factor inhibit sympathohippocampal sprouting. Brain Res Bull 15:639-634.

Toumane A, Durkin TP (1993) Time gradient for post-test vulnerability to scopolamine-induced amnesia following the initial acquisition session of a spatial reference memory task in mice. Behav Neural Biol 60:139-151.
Urschel BA, Hulsebosch CE (1992) Distribution and relative density of p75 nerve growth factor receptors in the brain as a function of age and treatment with antibodies to nerve growth factor. Brain Res 591:223-238.

Van-der-Zee C, Lourenssen S, Stanisz J, Diamond J (1995) NGF deprivation of adult rat brain results in cholinergic hypofunction and selective impairments in spatial learning. Eur J Neurosci 7:160-168.

Vantini G, Schiavo N, DiMartino A, Polato P, Triban C, Callegaro L, Toffano G, Leon A (1989) Evidence for a physiological role of nerve growth factor in the central nervous system of neonatal rats. Neuron 3:267-273.

Vazquez ME, Ebendal T (1991) Messenger RNAs for trk and the lowaffinity NGF receptor in rat basal forebrain. NeuroReport 2:593-596.

Yamamoto T, Matsuo R, Kawamura Y (1980) Localization of cortical gustatory area in rats and its role in taste discrimination. J Neurophysiol $44: 440-454$. 